\title{
Gliflozin bei Diabetes schützt auch Nieren
}

Empagliflozin schützt nicht nur die großen Gefäße sondern auch die Nieren von Typ-2-Diabetes-Patienten. So manifestiert sich ein Nierenschaden nicht nur deutlich seltener, auch sein Fortschreiten wird gebremst, wenn der SGLT-2-Hemmer zusätzlich zur Standardtherapie eingesetzt wird.

Nach und nach werden immer mehr Daten aus der Studie EMPA-REG OUTCOME ${ }^{\circledR}$ publiziert: beim diesjährigen Kongress der amerikanischen Diabetesgesellschaft unter anderem Details zur Nierenprognose mit Empagliflozin [1]. Prof. Christoph Wanner, Nephrologe an der Uniklinik Würzburg und Erstautor der Analyse der Nierendaten, präsentierte sie in New Orleans erstmals dem Publikum, das mit rauschendem Applaus reagierte.

Zu Erinnerung: Es handelt sich bei EMPA-REG OUTCOME ${ }^{\bullet}$ um eine randomisiert doppelblinde Studie über im Mittel 3 Jahre mit 7.020 Typ-2-Diabetikern mit hohem kardiovaskulärem Risiko. Sie erhielten 10 oder $25 \mathrm{mg} / \mathrm{d}$ Empagliflozin (Jardiance ${ }^{\circ}$ ) oder Placebo zusätzlich zur Standardtherapie (Blutdruck- und -fettsenkung, Plättchenhemmung). Patienten mit einer eGFR $<30 \mathrm{ml} / \mathrm{min} / 1,73 \mathrm{~m}^{2}$ waren ausgeschlossen. Ein Hauptergebnis: Das kardiovaskuläre Mortalitätsrisiko wurde um 38\% gesenkt.

\section{Nierenrisiko deutlich verringert}

Als ein präspezifizierter sekundärer Endpunkt war die Manifestation oder Verschlechterung einer Nephropathie festgelegt worden, definiert wie folgt: Progression zur Makroalbuminurie $>300 \mathrm{mg} / \mathrm{g}$ oder Verdoppelung des Serumkreatinins (begleitet von einem eGFR-Abfall $\leq 45 \mathrm{ml} / \mathrm{min} / 1,73 \mathrm{~m}^{2}$ ) oder Nierenersatztherapie oder aber Tod durch Niereninsuffizienz. Die eGFR lag zu Beginn im Mittel bei etwa $74 \mathrm{ml} / \mathrm{min} / 1,73 \mathrm{~m}^{2}$, ein Viertel der Teilnehmer hatte bereits eine Niereninsuffizienz (eGFR $<60$ ), etwa ein Drittel eine Albuminurie. Die Kontrolle erfolgten alle 3 Monate. Die relative Risikoreduktion für die genann-

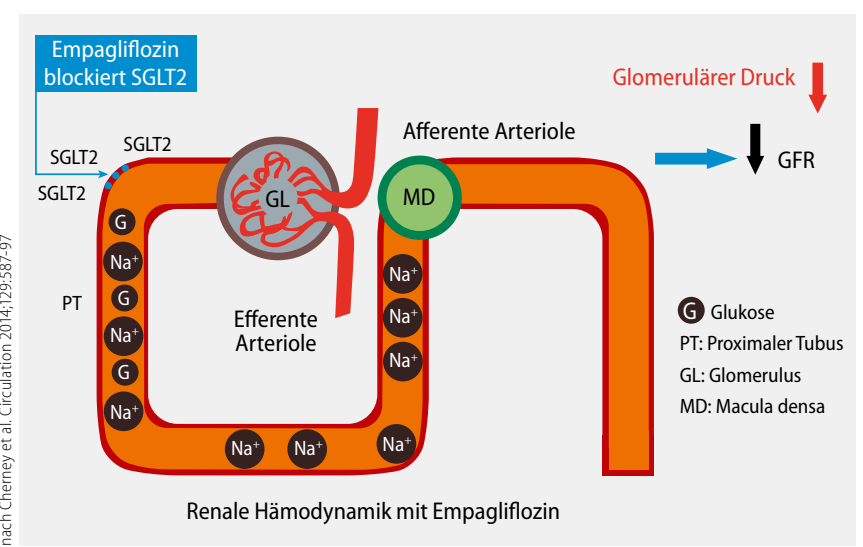

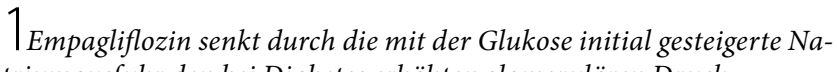
triumausfuhr den bei Diabetes erhöhten glomerulären Druck. ten Nierenereignisse innerhalb der Beobachtung von 3 Jahren lag mit Empagliflozin versus Placebo bei 39\% (95\%-KI 0,53$0,70, p<0,001)$. Eingetreten waren sie bei $12,7 \%$ mit dem Verumpräparat und 18,8\% mit Placebo. Die Dosierung des Gliflozins machte keinen Unterschied. Bei jenen, die schon bei Studienstart niereninsuffizient waren, wurde das Risiko einer weiteren Verschlechterung durch das Gliflozin um relative $42 \%$ gesenkt (95\%-KI 0,47-0,71, p <0,001).

Der Effekt war laut Wanner vorrangig getrieben von der verringerten Inzidenz der Makroalbuminurie. Allerdings habe der Schutz früh eingesetzt und kontinuierlich angehalten, was für den weiteren Verlauf in Bezug auf die härteren Endpunkte hoffen lasse. Mit Fokus auf die Verdopplung des Serumkreatinins, Nierenersatztherapie und Nierentod divergierten die Kurven erstmals nach etwa 1,5 Jahren (46\%ige Risikoreduktion HR 0,54 $(95 \%$-KI $0,40-0,75) \mathrm{p}<0,001)$.

\section{Gliflozin senkt glomerulären Hochdruck}

Während sich die Nierenfunktion in der Placebogruppe kontinuierlich verschlechterte, blieb sie in den beiden Verumgruppen nach initial leichtem eGFR-Abfall weitgehend stabil. Wanner erklärte diesen Verlauf mit dem Wirkmechanismus von Empagliflozin. Die SGLT-2-Hemmung stelle das glomeruläre Feedback über die Makula densa wieder her, indem das mit der Glukose zunächst vermehrt im Urin ausgeschiedene Natrium $\left(\mathrm{Na}^{+}\right)$die Adenosinsekretion forciert.

Die daraufhin erfolgende Engstellung des Vas afferens reduziert den bei Diabetikern erhöhten intraglomerulären Druck und so die Filtration ( $\triangleright$ Abb. 1) und Albuminurie. Wanner hob hervor, dass der Schutzeffekt den Subgruppenanalysen standhielt: Es funktioniert auch in fortgeschrittenen Stadien der Niereninsuffizienz, bei Patienten mit und ohne Albuminurie, Patienten unter und über 65 Jahren, bei Männern wie Frauen, bei Diabetesdauer von weniger als 5 oder mehr als 10 Jahren und bei $\mathrm{HbA}_{1 c}$-Werten über und unter $8,5 \%$. Und auch unabhängig von Adipositas, Hypertonie und der Basismedikation.

\section{Gute Verträglichkeit}

Bei den möglichen unerwünschten Effekten war kein neues Signal zu erkennen, kein Hinweis auf eine Steigerung der Hyperglykämie-, Fraktur- oder Ketoazidoserate und auch nicht für eine Steigerung des akuten Nierenversagens.

Die Sicherheit und Verträglichkeit war laut Wanner bei Patienten mit chronischer Niereninsuffizienz genauso gut wie in der Gesamtpopulation der Studie. Die Inzidenz der diabetischen Ketoazidose lag bei maximal 0,1\% und war in allen Behandlungsgruppen vergleichbar.

Sarah Louise Pampel

Literatur:

1. Wanner C, Inzucchi SE, Lachin JM et al. Empagliflozin and Progression of Kidney Disease in Type 2 Diabetes. N Engl J Med. 2016 Jul 28;375(4):323-34.

Quelle: Symposium „Update from the Empa-Reg Outcome Trial”, 14.6.2016, 76th Scientific Sessions der American Diabetes Association in New Orleans 